\title{
Effect of ketyl radical on the structure and performance of holographic polymer/liquid-crystal composites
}

\author{
Xiaoyu Zhao ${ }^{1}$, Shanshan Sun ${ }^{2}$, Ye Zhao ${ }^{1}$, Rong-Zhen Liao ${ }^{1}$, Ming-De Li ${ }^{2 *}$, Yonggui Liao ${ }^{1}$, \\ Haiyan Peng ${ }^{1 *}$ and Xiaolin Xie ${ }^{1}$
}

\begin{abstract}
Holographic polymer/liquid-crystal composites, which are periodically ordered materials with alternative polymer-rich and liquid-crystal-rich phases, have drawn increasing interest due to their unique capabilities of reconstructing colored three-dimensional (3D) images and enabling the electro-optic response. They are formed via photopolymerization induced phase separation upon exposure to laser interference patterns, where a fast photopolymerization is required to facilitate the holographic patterning. Yet, the fast photopolymerization generally leads to depressed phase separation and it remains challenging to boost the holographic performance via kinetics control. Herein, we disclose that the ketyl radical inhibition is able to significantly boost the phase separation and holographic performance by preventing the proliferated diffusion of initiating radicals from the constructive to the destructive regions. Dramatically depressed phase separation is caused when converting the inhibiting ketyl radical to a new initiating radical, indicating the significance of ketyl radical inhibition when designing high performance holographic polymer composites.
\end{abstract}

Keywords: liquid crystal, ordered structures, photopolymerization, inhibition, holography

\section{INTRODUCTION}

Holography is a powerful technique that encodes information via the wave field interference [1]. Due to its unique capability of simultaneously reconstructing the whole information of coherent waves (e.g., amplitude, phase and polarization), holography has become a con- stant innovation source in ultrafast temporal imaging $[2,3]$, optical shaping [4,5], particle assembly [6,7], threedimensional (3D) display $[8,9]$, colored 3D image storage [10-16], and holographic polymer electrolyte construction [17]. On the other hand, polymer/liquid-crystal (LC) composites have attracted considerable attention due to their unique electro-optic response capability [18-21]. Integrating holography and polymer/LC composites results in the generation of holographic polymer/LC composites, which not only allows for the reconstruction of holographic images but also provides attractive electrooptic response [22-25].

Holographic polymer/LC composites are periodicallyordered polymer/LC domains with alternative polymerrich and LC-rich phases. They are formed via photopolymerization induced phase separation upon exposure to laser interference patterns. For improving the phase separation and holographic performance, it is critical to exert a spatiotemporal control over the photopolymerization kinetics and gelation. The fast gelation process in the constructive regions allows for a rapid holographic patterning while the prolonged gelation in the destructive regions provides longer time for the phase separation [12-14]. Nevertheless, despite the amazing progress on the elegant design of new photo-mediated systems [26-36] for a myriad of attractive applications such as sequential polymerization control [29], 3D printing [30], surface grafting [31], and fabricating conductive composites [36], very few efforts have been made to implement such a spatiotemporal control during holography [13]. Peng and co-workers $[12,13]$ disclosed that

\footnotetext{
${ }^{1}$ Key Lab for Material Chemistry of Energy Conversion and Storage, Ministry of Education, School of Chemistry and Chemical Engineering, and National Anti-Counterfeit Engineering Research Center, Huazhong University of Science and Technology (HUST), Wuhan 430074, China

${ }^{2}$ Department of Chemistry and Key Laboratory for Preparation and Application of Ordered Structural Materials of Guangdong Province, Shantou University (STU), Shantou 515063, China

*Corresponding authors (emails: mdli@stu.edu.cn (Li MD); hypeng@hust.edu.cn (Peng H))
} 
a "photoinitibitor" with concurrent initiation and inhibition functions was able to exert a precise control over the photopolymerization kinetics and gelation during holographic patterning. The ketyl radical generated from the "photoinitibitor" may play a critical role. However, the effect of ketyl radical on the performance of holographic polymer/LC composites is insufficiently understood. Herein, we intend to understand the effect of ketyl radical on the structure and performance of holographic polymer/LC composites by eliminating the ketyl radical without affecting the primary photoreaction of the "photoinitibitor".

It is reported that the ketyl radical could be scavenged by 2-(4-methoxyphenyl)-4,6-bis(trichloromethyl)-1,3,5triazine (TA) [37,38]. Thus, we are inspired to add TA into the "photoinitibitor" composed of 3,3'-carbonylbis (7-diethylaminocoumarin) (KCD) and $\mathrm{N}$-phenylglycine (NPG) (Scheme 1). Interestingly, TA successfully converts the inhibiting ketyl radical to a new initiating radical without exhibiting a detectable influence on the primary photoreaction of KCD with NPG. Because of the unique inhibition function of the ketyl radical, dramatically improved holographic polymer/LC composites with a high diffraction efficiency $(\eta=93 \pm 4 \%)$ are enabled. Dramatically depressed phase separation and holographic performance $(\eta=11 \pm 4 \%)$ are caused when converting the inhibiting ketyl radical to a new initiating radical, indicating the significance of ketyl radical inhibition on the regulation of holographic polymer composites.

\section{EXPERIMENTAL}

\section{Materials}

N,N-dimethylacrylamide (DMAA, purity: 98\%), 5,5-dimethyl-1-pyrroline $N$-oxide (DMPO, purity: 97\%), TA (purity: 98\%) were purchased from TCI Chemicals. 2-Ethylhexyl acrylate (EHA, purity: $98.5 \%$ ), N,Ndimethylformamide (DMF, purity: AR), ethyl acetate (EA, purity: AR), hexane (purity: AR), toluene (spectrometric grade, purity: 99.7\%), tetrahydrofuran (THF, spectrometric grade, purity: 99.9\%), NPG (purity: 97\%), and acetonitrile (purity: AR) were received from Aladdin. KCD (purity: 99\%) was obtained from Acros Organics. The nematic liquid crystal mixture P0616A $\left(n_{\mathrm{o}(589 \mathrm{~nm}, 293 \mathrm{~K})}=\right.$ $\left.1.52, n_{\mathrm{e}(589 \mathrm{~nm}, 293 \mathrm{~K})}=1.72, T_{\mathrm{N} 1}=331 \mathrm{~K}\right)$ [39] was purchased from Shijiazhuang Chengzhi Yonghua Display Material Co., Ltd., China. The hyperbranched acrylate monomer 6361-100 (functionality: 8) was donated as a gift by Eternal Chemical Co., Ltd., China. NPG was recrystallized from hot water in the presence of activated charcoal before use, and other chemicals were used directly without further purification. The chemical struc-
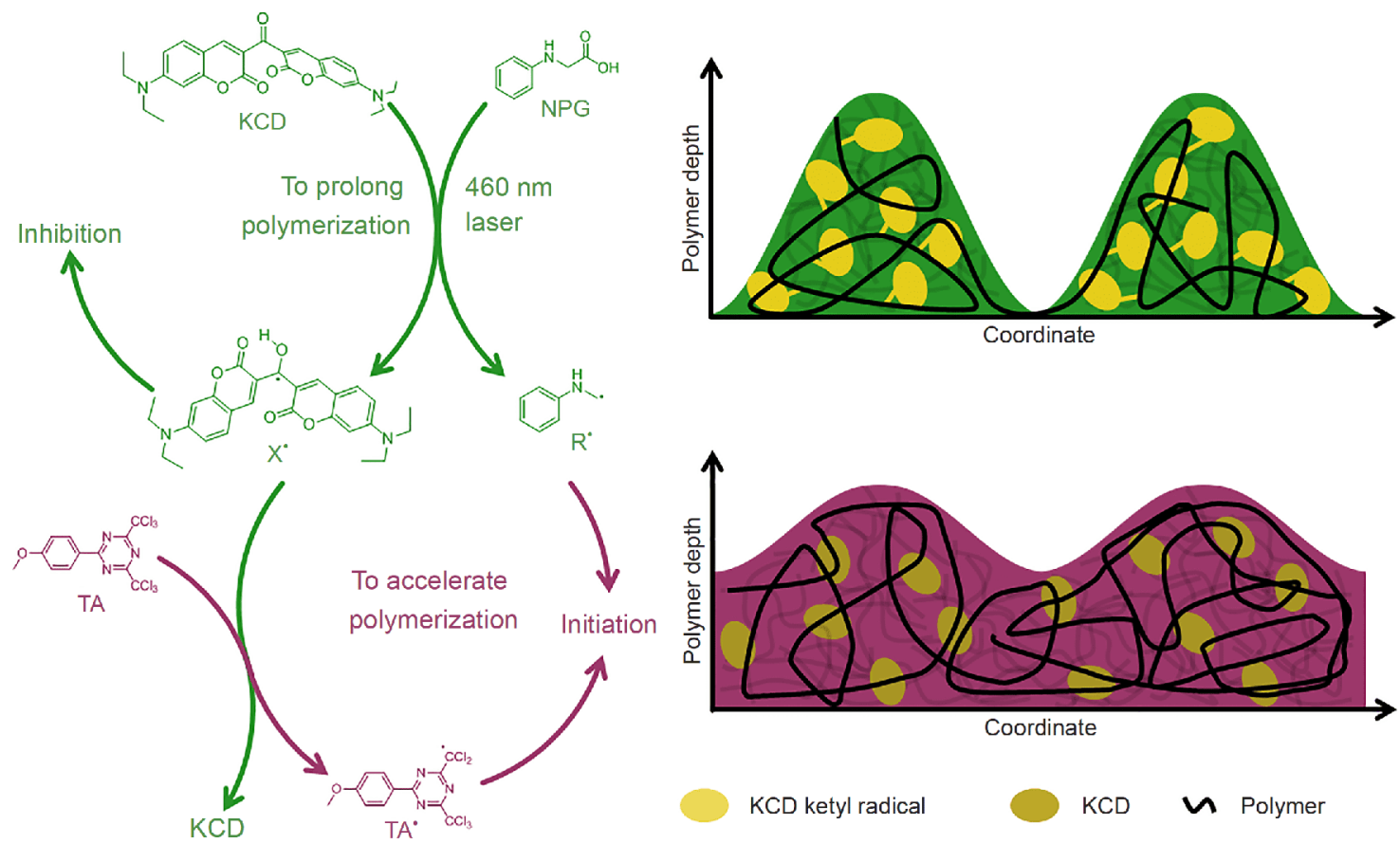

Scheme 1 Schematic illustration on the conversion of the ketyl radical with an inhibition function to a new initiating radical and the corresponding ordered structures. 
tures of KCD, NPG, TA, 6361-100, EHA and DMAA are displayed in Scheme 2.

\section{Photo-mediated copolymerization of DMAA and EHA}

To understand the inhibition mechanism of the ketyl radical, the resulting photopolymer was analyzed. The solid KCD and NPG were dissolved in the DMAA/EHA mixture (weight ratio: 1/1) to form homogeneous solutions upon bulk ultrasonication for $30 \mathrm{~min}$ at $323 \mathrm{~K}$. DMAA helped to dissolve the KCD and NPG, while EHA was used to boost the photopolymerization. The concentration of KCD and NPG was controlled to be 6 and $60 \mathrm{mmol} \mathrm{L}^{-1}$, respectively. The solution was injected into a glass cell (gap thickness: $50 \mu \mathrm{m}$ ) and then irradiated by a $2 \mathrm{~mW} \mathrm{~cm}^{-2}$ of $460 \mathrm{~nm}$ laser light for $60 \mathrm{~min}$. After photopolymerization, the copolymer was scratched off from the glass slide, and then precipitated from the EA/hexane (volume ratio: 1/50) mixture, finally dried under vacuum overnight to remove the residual solvents.

\section{Ultraviolet-visible (UV-vis) absorption}

The ground state absorption was characterized using a UV-vis spectrophotometer (Evolution 220, Thermo Fisher). Before characterization, the chemicals were dissolved in DMF or acetonitrile by ultrasonication at room temperature to form homogenous solutions.

\section{Nanosecond transient absorption}

To understand the reaction mechanism, the nanosecond transient absorption of KCD, KCD/NPG, KCD/NPG/TA was carefully explored. Different from our previous report [13], herein the nanosecond transient absorption measurements were performed in the flowing mode. To balance the signal intensity and accuracy, the concentrations of KCD, NPG and TA were optimized to be $175 \mu \mathrm{mol} \mathrm{L}^{-1}$, separately. The pump wavelength was $400 \mathrm{~nm}$ so that only KCD was excited while avoiding the excitation of NPG and TA. The solution was purged with dry nitrogen gas for $30 \mathrm{~min}$ in dark before irradiation and continuously purged during measurements.

\section{Electron paramagnetic resonance (EPR) spectroscopy}

To identify the radical species, the EPR analysis was implemented. Homogeneous solutions of KCD/NPG and $\mathrm{KCD} / \mathrm{NPG} / \mathrm{TA}$ in toluene were carefully loaded in the columnar quartz cell for EPR analysis, respectively. The concentrations of KCD, NPG and TA were $175 \mu \mathrm{mol} \mathrm{L}^{-1}$ for EPR measurement, respectively, consistent with that for nanosecond transient absorption characterization. The solution was extensively purged with dry nitrogen gas for $30 \mathrm{~min}$, followed by excitation inside the EPR spectrometer cavity with the mercury lamp equipped with a $420-1100 \mathrm{~nm}$ light filter. Since the TA and purified NPG showed no absorption above the wavelength of $380 \mathrm{~nm}$, they were not photoexcited under such condition. EPR spectra were recorded on a Bruker EMXmicro spectrometer. Following settings were applied for the measurement: center field, 3500 Gauss (G); sweep width, $100 \mathrm{G}$; microwave power, $2 \mathrm{~mW}$; modulation frequency, $100 \mathrm{kHz}$; modulation amplitude, $1 \mathrm{G}$; receiver gain, $30 \mathrm{~dB}$; conversion time, $15 \mathrm{~ms}$; time constant, $0.01 \mathrm{~ms}$. A radical capturing agent 5,5-dimethylpyrroline $\mathrm{N}$-oxide (DMPO, $3.5 \mathrm{mmol} \mathrm{L}^{-1}$ ) was introduced to stabilize the radicals during characterization. Signals were fitted with the EasySpin software [40] to identify the radical species.

\section{Holographic formulation}

The holographic mixture was mainly composed of DMAA, 6361-100 and P0616A in a weight ratio of 14:7:9. Since a multifunctional monomer with low viscosity is important for regulating the grating structure and improving the holographic performance, we employed 6361-100 as the multi-functional monomer as previously optimized [41]. For the "photoinitibitor" system, KCD and NPG were homogeneously dissolved in the holographic mixture upon bulk ultrasonication at $323 \mathrm{~K}$ for
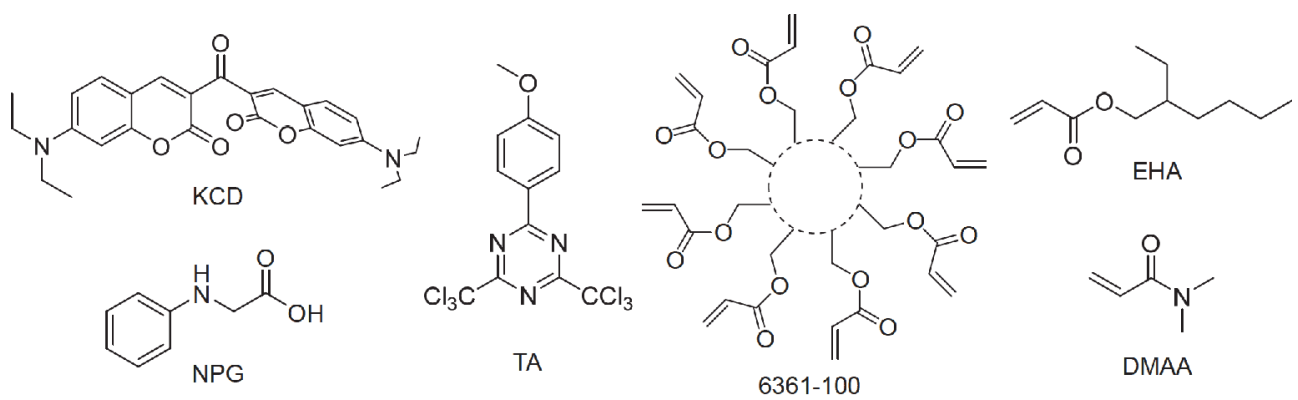

Scheme 2 Chemical structures of KCD, NPG, TA, 6361-100, EHA and DMAA. 
$1 \mathrm{~h}$. A system with TA was also prepared using the same method. The concentration of KCD was controlled to be $6 \mathrm{mmol} \mathrm{L}^{-1}$ while those of NPG and TA were kept at $60 \mathrm{mmol} \mathrm{L}^{-1}$. Brown-color vials with sealed caps were used during the formulation process to prevent any photoreaction.

\section{Photopolymerization kinetics}

The photopolymerization kinetics were analyzed using real-time Fourier transform infrared spectroscopy (RTFTIR, Vertex 80, Bruker). A narrow-band pass filter centered at $460 \mathrm{~nm}$ was used to confine the light irradiation (S2000, Omnicure). The light intensity was set as $3.0 \mathrm{~mW} \mathrm{~cm}^{-2}$. Considering the light transmittance (86.6\%) of $\mathrm{NaCl}$ plates (thickness: $4 \mathrm{~mm}$ ) at $460 \mathrm{~nm}$ (Fig. S1), the light intensity reaching the sample was $2.6 \mathrm{~mW} \mathrm{~cm}^{-2}$. The holographic mixture was placed between two $\mathrm{NaCl}$ plates with a $20 \mu \mathrm{m}$-thick spacer. The absorption peaked at $950 \mathrm{~cm}^{-1}$ was used to monitor the conversion of $\mathrm{C}=\mathrm{C}$ double bond during photopolymerization, which was assigned to the rocking vibration of vinyl groups (Figs S2-S4). The double bond conversion $(\alpha)$ and photopolymerization rate $\left(R_{\mathrm{p}}\right)$ were calculated as follows [42],

$\alpha=1-\frac{A_{t}}{A_{0}}$,

$R_{\mathrm{p}}=\frac{\mathrm{d} \alpha}{\mathrm{d} t}$,

where, $A_{\mathrm{t}}$ and $A_{0}$ represented the absorption areas peaked at $950 \mathrm{~cm}^{-1}$ at the irradiation time $t$ and before light irradiation, respectively.

\section{Photorheology}

To characterize the gelation behavior of holographic mixtures, a rheometer (MCR 302, Anto-Parr) with a light accessory (S2000, Omnicure) was employed. The sample thickness was maintained to be $0.1 \mathrm{~mm}$ by two parallel circular plates (diameter: $25 \mathrm{~mm}$ ). The above one was rotatable and connected to the detector, while the bottom one was still and transparent so that the light could go through to fully cure the holographic mixture. The measurement was conducted at $303 \mathrm{~K}$ under the protection of nitrogen gas purge $\left(50 \mathrm{~mL} \mathrm{~min}^{-1}\right)$. One narrowband pass filter centered at $460 \mathrm{~nm}$ was employed to confine the irradiation beams. The light intensity was set as $3.0 \mathrm{~mW} \mathrm{~cm}{ }^{-2}$. Continuous shearing for $60 \mathrm{~s}$ was performed prior to photorheology test. The crossover of storage modulus and loss modulus was regarded as the gel point $[43,44]$.

\section{Holographic patterning}

One $460 \mathrm{~nm}$ laser was used to reconstruct the holographic polymer/LC composites. Uniform grating ordered structures were first recorded through the interference of two coherent beams which were generated by splitting the original laser. The bisector of the two beams was normal to the surface plane so that unslanted grating ordered structures could be formed. Before holographic patterning, the holographic mixture was injected into an ITO coated glass cell in dark, and then kept still on the sample holder for $60 \mathrm{~s}$. The sample thickness was controlled to be $10 \mu \mathrm{m}$. The light intensity was set as $3.0 \mathrm{~mW} \mathrm{~cm}^{-2}$ for each beam and the exposure time was $30 \mathrm{~s}$. The grating period $(\Lambda)$ was calculated to be $889 \mathrm{~nm}$ according to the Bragg's law [41],

$\Lambda=\frac{\lambda_{\text {writing }}}{2 \sin \frac{\theta_{\text {set }}}{2}}$,

where, $\lambda_{\text {writing }}$ was the wavelength of the writing laser, and $\theta_{\text {set }}$ was the angle between the two coherent laser beams $\left(30^{\circ}\right)$, respectively.

To reconstruct holographic images, a spatial light modulator (SLM) was used to project the computergenerated image into the inference field as the object beam. Images were reconstructed in the holographic polymer/LC composites once another reference beam directly irradiated the sample and interfered with the object beam. To solidify the structures, all samples were postcured upon the UV flood irradiation for $10 \mathrm{~min}$.

\section{Morphology characterization}

The morphology of holographic polymer/LC composites was characterized using field-effect scanning electron microscopy (FE-SEM, Sirion 200) and atomic force microscopy (AFM, Shimadzu SPM-9700). Samples were immersed in $n$-hexane for $48 \mathrm{~h}$ to remove the LC prior to characterization, so that the phase separated structures were easily recognized. AFM characterization was implemented in the tapping mode (resonant frequency: $300 \mathrm{kHz}$ ) after drying the sample under ambient atmosphere. A thin layer of platinum was sputtered on the top surface of the sample prior to SEM measurement to enhance the image contrast.

\section{Electro-optic response}

A p-polarized $633 \mathrm{~nm}$ laser $(5 \mathrm{~mW})$ was employed to nondestructively probe the holographic polymer/LC composites at the Bragg angle. The diffraction efficiency $(\eta)$ was defined as the ratio of the diffraction intensity $\left(I_{\mathrm{D}}\right)$ to the total intensities of both diffraction and transmis- 
sion beams $\left(I_{\mathrm{T}}\right)$ at the Bragg angle (Equation (4), Scheme 3). During characterization, a display parameter tester (LCT-5016C, North LC Engineering Research and Development Center, China) was utilized to apply a square alternating current (AC) voltage (amplitude: $28 \mathrm{~V} \mathrm{\mu m}^{-1}$, frequency: $1 \mathrm{kHz}$ ) to drive the holographic polymer/LC composites.

$\eta=\frac{I_{\mathrm{D}}}{I_{\mathrm{D}}+I_{\mathrm{T}}}$.

\section{Computational details}

Density functional theory (DFT) calculations were performed using the M06-2X hybrid functional with the Gaussian 09 software [45]. The structures were optimized using the $6-311 \mathrm{G}(\mathrm{d}, \mathrm{p}$ ) basis set (Table S1-S4). Analytic frequency calculations were performed to confirm the nature of the stationary points (no imaginary frequency). The spin density was calculated using the $6-311 \mathrm{G}(\mathrm{d}, \mathrm{p})$ basis set with the polarizable continuum model (PCM, toluene).

The structure of the KCD first singlet excited state $\left(\mathrm{S}_{1}\right)$ was optimized at the M06-2X/6-311G(d,p) level using the time-dependent DFT (TD-DFT) calculations (Table S5).

The UV-vis spectra in toluene were calculated at the M06-2X/6-311+G(d,p) level using the PCM model. All calculated spectra were scaled by 1.23 times. To reduce the computational cost, the structures of KCD and the radical coupling product of ketyl radical were simplified as M-KCD and M-KCD', respectively (Scheme 4).

\section{RESULTS AND DISCUSSION}

\section{Ground state absorptions of KCD, NPG and TA}

To precisely control the photoreactions of the KCD/NPG "photoinitibitor" and the KCD/NPG/TA system, it is critical to photoexcite the KCD rather than NPG or TA. Toward this end, the ground state absorptions of KCD, NPG and TA were characterized, respectively. Clearly, KCD shows a peak absorption at $460 \mathrm{~nm}$ with an ex-

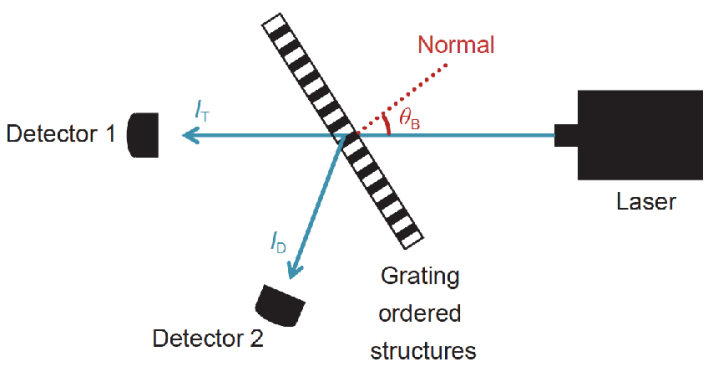

Scheme 3 Schematic illustration on the diffraction efficiency measurement.

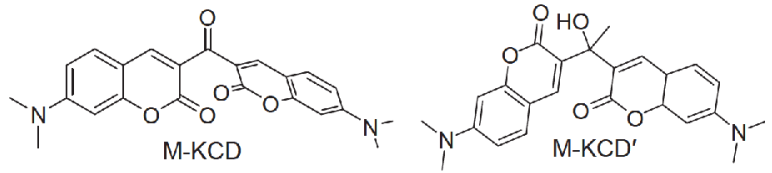

Scheme 4 Simplified structures of KCD and the radical coupling product of ketyl radical for calculations.

tinction coefficient of $8.8 \times 10^{4} \mathrm{~L} \mathrm{~mol}^{-1} \mathrm{~cm}^{-1}$ (Fig. 1), which is expected to facilitate the holographic patterning upon the $460 \mathrm{~nm}$ laser irradiation. By contrast, no absorption is noted for the purified NPG or TA above the wavelength of $380 \mathrm{~nm}$.

\section{Transient absorption of excited KCD}

To understand the photoreaction of KCD with NPG, we firstly recorded the transient absorption of pure KCD in toluene after excitation by $400 \mathrm{~nm}$ pump light. As shown in Fig. 2a, a positive absorption band peaked at $490 \mathrm{~nm}$ arises at $\sim 0.5 \mathrm{~ns}$ upon the $400 \mathrm{~nm}$ pulse laser irradiation, which is ascribed to the KCD $S_{1}$ state according to TDDFT calculations (Fig. 2d and Fig. S5). A negative absorption band from 410 to $472 \mathrm{~nm}$ is also observed, which is caused by the ground-state bleach of KCD. With time going on, the positive peak absorption continues to increase and shifts from 490 to $515 \mathrm{~nm}$ after $\sim 0.8 \mathrm{~ns}$ (Fig. 2b), indicating an intersystem crossing (ISC) from the $\mathrm{S}_{1}$ to the first triplet excited state $\left(\mathrm{T}_{1}\right.$, Fig. $2 \mathrm{~d}$ and Fig. S5) [46]. Finally, both negative and positive signals shift to zero from $\sim 1.5 \mathrm{~ns}$ (Fig. $2 \mathrm{c}$ ), indicating a decay of the $\mathrm{KCD} \mathrm{T}_{1}$ state.

\section{Transient absorption evidence for the KCD derived ketyl radical}

It is well known that an aromatic ketone holds a high

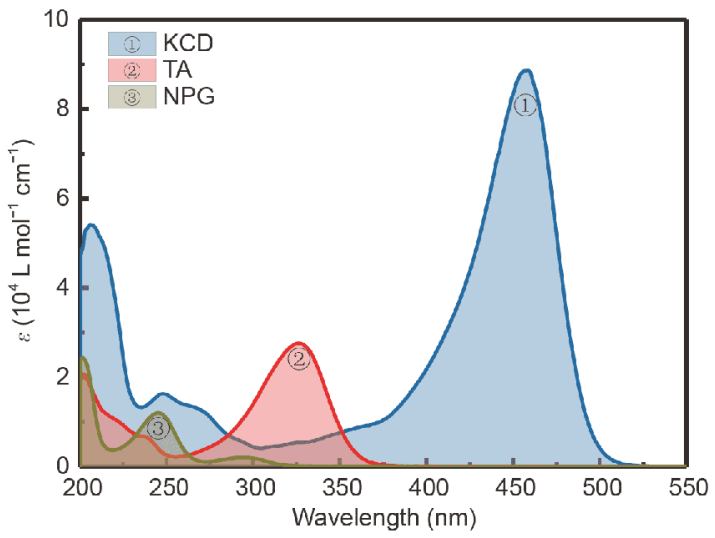

Figure 1 Ground state absorptions of KCD, NPG and TA in acetonitrile. NPG was recrystallized before use. 

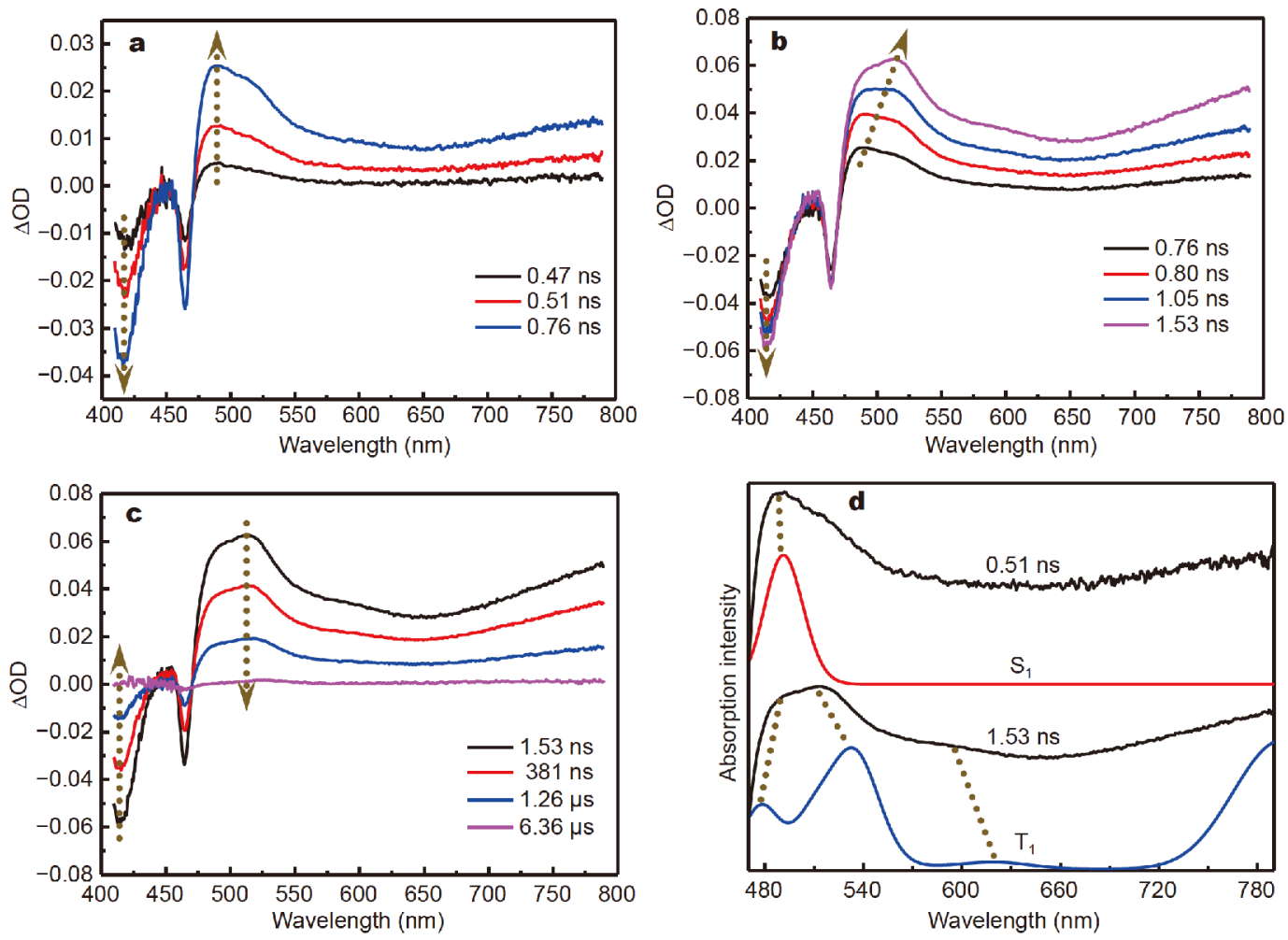

Figure $2(\mathrm{a}-\mathrm{c})$ Nanosecond transient absorption of pure $\mathrm{KCD}\left(175 \mu \mathrm{mol} \mathrm{L}^{-1}\right)$ in toluene upon a $400 \mathrm{~nm}$ pulse laser excitation. (d) Comparison between the experimental spectra (black line) and TD-DFT calculations (red and blue lines). The calculated spectra are scaled by 1.23 times with a half-width of $700 \mathrm{~cm}^{-1}$.

triplet population upon light irradiation, since the lowlying lone pair electrons on the oxygen $p$ orbital facilitate the hybridization of $n-\pi^{*}$ and $\pi-\pi^{*}$ transitions, although no heavy atoms exist. Nevertheless, despite that a diphenyl ketone usually holds a high energy $T_{1}$ state to grab one hydrogen atom directly from other compounds (e.g., alkyl substituted compounds) upon UV irradiation $[35,47,48]$, the triplet energy of ketocoumarin (e.g., KCD) is not high enough to grab one hydrogen atom directly from other compounds [46]. Therefore, only the coinitiator with a low oxidation potential (e.g., NPG) can react with the excited KCD via proton coupled electron transfer (PCET) $[13,46]$.

When irradiating the KCD/NPG "photoinitibitor" in toluene by a $400 \mathrm{~nm}$ pulse laser, NPG is not excited and also does not influence the formation of $\mathrm{KCD} \mathrm{S}_{1}$ state and the ISC process from the $S_{1}$ to $T_{1}$ state (Fig. 3a). Nevertheless, after $\sim 2.4 \mathrm{~ns}$, a red shift of the peak absorption from $515\left(\mathrm{~T}_{1}\right)$ to $528 \mathrm{~nm}$ is observed (Fig. 3b), indicating the formation of ketyl radical [13], which is further supported by the TD-DFT calculation (Fig. 3d). A decreasing of transient absorption is noted when further increasing the monitoring time (Fig. 3c), indicating the decay of ketyl radical. It is worth noting that the triplet KCD holds a long lifetime $(1.04 \mu$ s, Fig. S6), which is caused by the spin forbidden from $T_{1}$ to $S_{0}$. The long lifetime of triplet KCD facilitates the electron and proton transfer from NPG to the KCD triplet state, which is expected to afford a high initiation rate during photopolymerization [13]. In addition, the lifetime of $\mathrm{KCD} \mathrm{T}_{1}$ state decreases to $0.68 \mu$ s owning to the PCET reaction with NPG. Interestingly, the lifetime of ketyl radical is $7.78 \mu \mathrm{s}$ from the biexponential fitting (Fig. S6), which is 7.7 times larger than that of triplet KCD. Because of the quite long lifetime, the ketyl radical can be detected by EPR and holds enough time to inhibit the photopolymerization.

\section{EPR evidence of the KCD derived ketyl radical}

To further confirm the formation of ketyl radical, EPR spectra were captured upon the visible light irradiation. NPG is not excited under such condition based on the UV-vis absorption spectrum of ground state (Fig. 1). A radical capturing agent DMPO was employed to stabilize the radicals produced and improve the EPR signal-tonoise ratio. As reported by Blount, Janzen and co-workers 

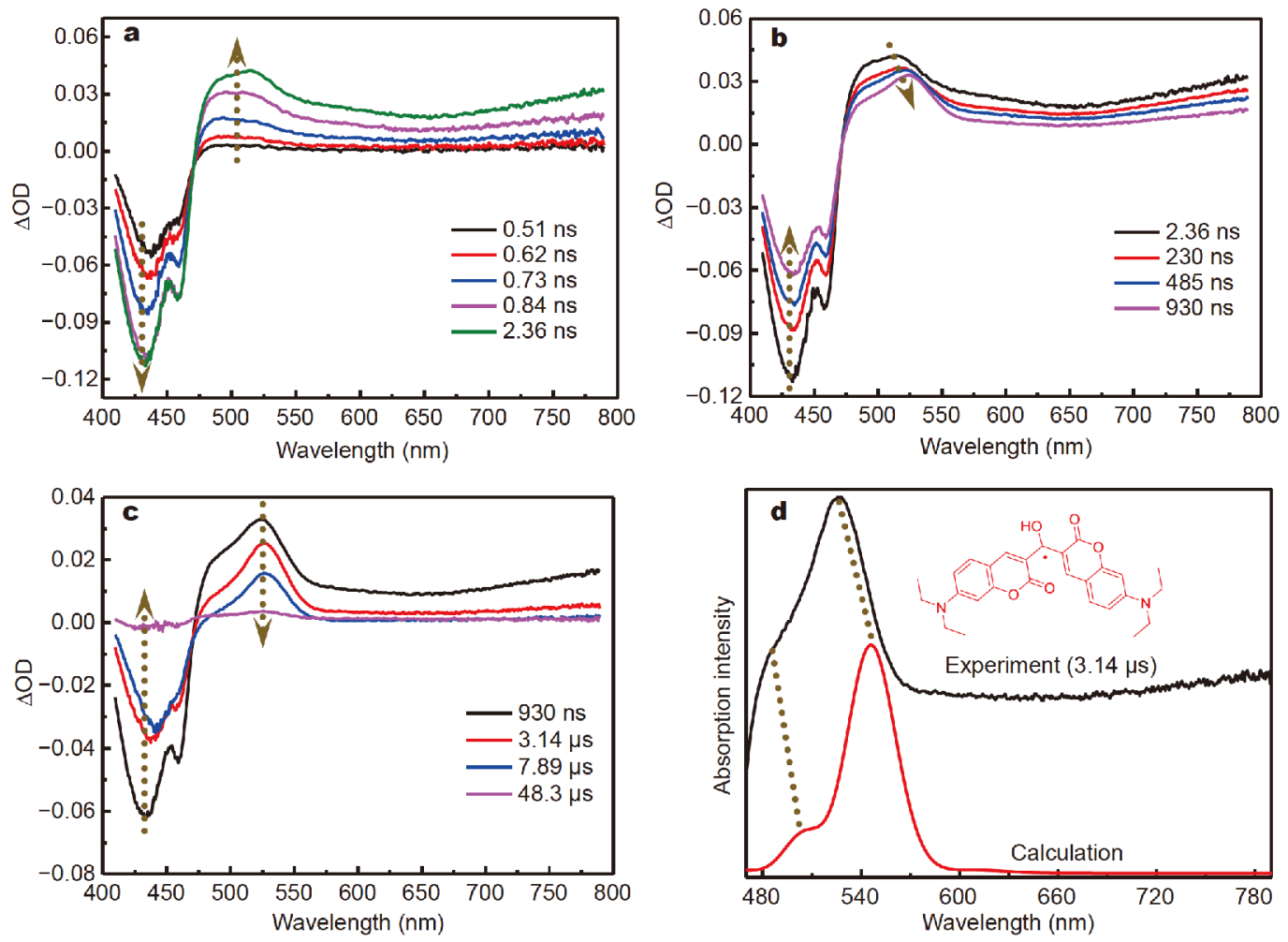

Figure 3 (a-c) Nanosecond transient absorption when irradiating the KCD/NPG "photoinitibitor" $\left(175 \mu\right.$ mol L ${ }^{-1}$ for each in toluene) by a $400 \mathrm{~nm}$ pulse laser. (d) Spectra comparison between the experimental result (black line) and TD-DFT calculation (red line). The calculated spectrum is scaled by 1.23 times with a half-width of $700 \mathrm{~cm}^{-1}$.

[49], the safe potential window of DMPO ranges from -1.8 to $1.3 \mathrm{~V}$ (versus saturated calomel electrode, namely $\mathrm{SCE})$. Clearly, DMPO is not ready to react with KCD or NPG, because both the potential of KCD $(-1.23 \mathrm{~V} v s$. SCE for reduction potential and $1.06 \mathrm{~V}$ vs. SCE for oxidation potential) and oxidation potential of NPG $(0.43 \mathrm{~V}$, vs. SCE) are in the safe potential window of DMPO [13].

After the photoreaction of triplet KCD with NPG, two radicals are produced, namely the ketyl radical and amino-alkyl radical, respectively. The KCD derived ketyl radical (radical 1, Fig. 4a) is stable to some extent because of the favorable electron delocalization across the large planar conjugated aromatic ring [13]. Thus, the ketyl radical possesses a relatively long lifetime $(\sim 7.78 \mu \mathrm{s})$ that can be detected by EPR. Meanwhile, the KCD derived ketyl radical can also react with DMPO, forming another radical (radical 2). At the same time, the amino-alkyl radical is not stable and favorable to react with the $\mathrm{C}=\mathrm{N}$ double bond of DMPO [50]. Thus, only the amino-alkyl radical captured by DMPO is detected (radical 3). The spin densities on radical 1 from our DFT calculations are mainly located at C1, C2, C3 and O4 (Fig. S7). By contrast, the spin densities on radicals 2 and 3 are located at the $\mathrm{N}$ and $\mathrm{O}$ atoms of the DMPO unit, as reported previously [51]. Thus, only the atoms related to these electron spin centers need to be considered when performing the EPR simulation. Interestingly, the simulated EPR signal matches well with the experimental data (Fig. 4b), indicating the main generation of ketyl radical and amino-alkyl radical during the photoreaction of KCD with NPG. The hyperfine coupling constants are $12.1 \mathrm{G}(\mathrm{H} 5)$ and $11.3 \mathrm{G}(\mathrm{H} 6)$ for radical 1 ( $g$-value, 2.0067); $11.5 \mathrm{G}$ $(\mathrm{H} 3)$ and $13.4 \mathrm{G}(\mathrm{N} 2)$ for radical 2 (g-value, 2.0062); $20.6 \mathrm{G}(\mathrm{H} 3)$ and $14.3 \mathrm{G}(\mathrm{N} 2)$ for radical 3 ( $g$-value, 2.0061 ), respectively, in good coincidence with the previous report [52].

\section{Inhibition of the propagating radical by the ketyl radical through direct coupling reaction}

The photo-generated ketyl radical shows a clear inhibition capability $[13,14,53,54]$, which is recently realized to be able to boost the spatiotemporal control over photopolymerization $[11,13,54]$. Nevertheless, the inhibition mechanism is still unclear. Toward this end, the copolymer P(DMAA-co-EHA) was prepared through the visible light photopolymerization mediated by the $\mathrm{KCD} / \mathrm{NPG}$ 

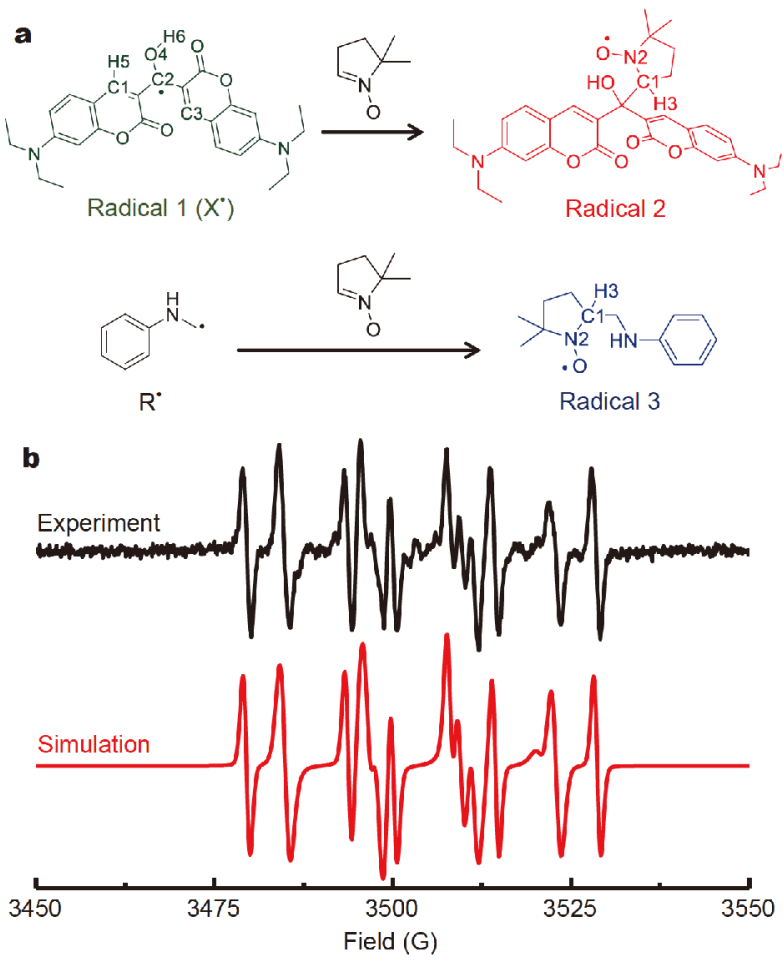

Figure 4 (a) Chemical structures of radicals 1, 2 and 3. (b) Experimental and simulated EPR signals of the KCD/NPG "photoinitibitor" upon visible light irradiation.

"photoinitibitor". DMAA helps the dissolution of KCD and NPG while EHA with a higher propagation constant facilitates the photopolymerization [55]. Interestingly, the purified P(DMAA-co-EHA) $\left(M_{\mathrm{n}}, 79 \mathrm{kDa}\right)$ in DMF shows a peak absorption at $397 \mathrm{~nm}$, which is blue shifted for $64 \mathrm{~nm}$ compared with that of pure KCD (Fig. 5), indicating that a coupling reaction between the ketyl radical and the macromolecular radical occurs. This interpretation is further supported by the TD-DFT calculations (Fig. 5). A control experiment was also implemented to further demonstrate the coupling reaction, where the $\mathrm{P}$ (DMAA-co-EHA) $\left(M_{\mathrm{n}}, 102 \mathrm{kDa}\right)$ was prepared upon UV irradiation by using NPG as the initiator. No absorption is observed in the wavelength range of $350-700 \mathrm{~nm}$ (Fig. S8), further confirming that the peak absorption at $397 \mathrm{~nm}$ is caused by the KCD derived ketyl radical.

\section{Converting the ketyl radical to a new initiating radical}

To understand the role of KCD ketyl radical during holographic photopolymerization, the inhibiting ketyl radical of the KCD/PNG "photoinitibitor" needs to be scavenged. TA is reported to be able to convert the inhibiting ketyl radical to a new initiating radical $[37,38]$,

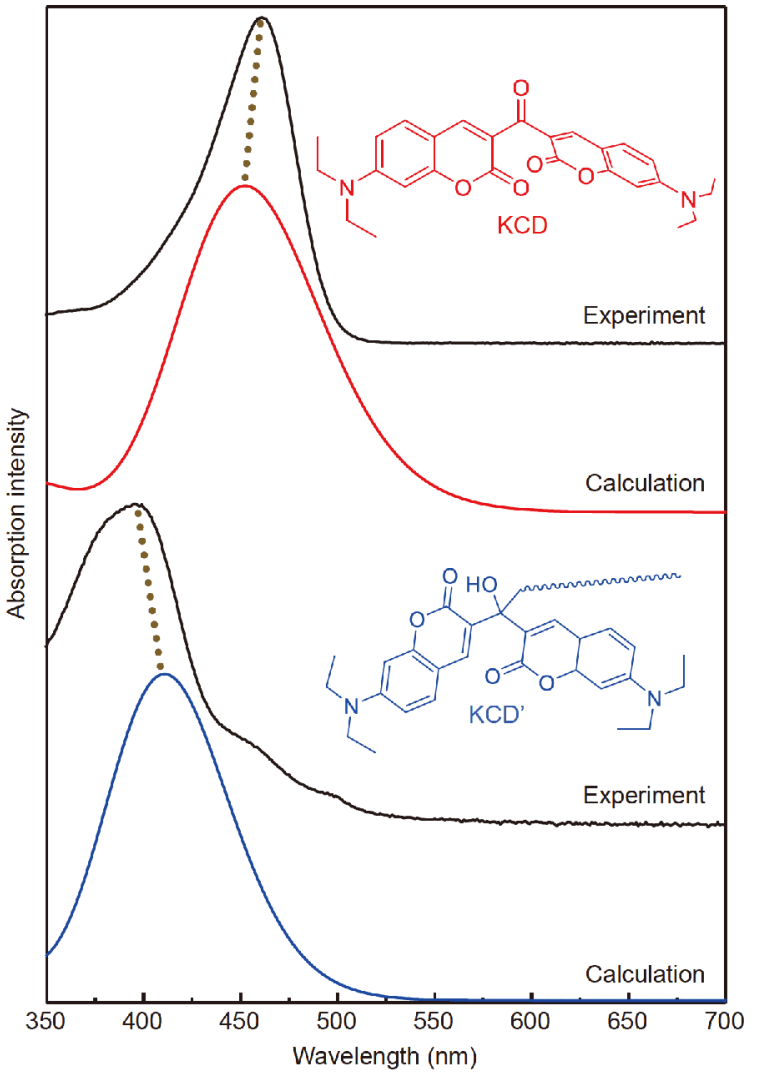

Figure 5 Comparison between the experimental and calculated absorptions of KCD and KCD' (the radical coupling product). DMF is the solvent during characterization. All alkyl chains are replaced with the methyl group during DFT calculations to save the computation resource. The calculated spectra are scaled by 1.23 times with a half-width of $2500 \mathrm{~cm}^{-1}$.

although the influence of TA on the primary photoreaction of KCD with NPG still remains unclear.

To scavenge the ketyl radical, one equivalent TA was added in the KCD/NPG mixture. As displayed in Fig. 6, the photoreaction process of KCD/NPG/TA can be separated into four steps: 1$) \mathrm{KCD}$ is photo-excited into its $\mathrm{S}_{1}$ state at $\sim 0.5 \mathrm{~ns}$ upon a $400 \mathrm{~nm}$ pulse laser irradiation. 2) The KCD $S_{1}$ state transforms into its $T_{1}$ state after $\sim 0.8 \mathrm{~ns}$. 3) The $\mathrm{KCD} \mathrm{T}_{1}$ state reacts with NPG, producing the ketyl radical and amino-alkyl radical after $\sim 2.4 \mathrm{~ns}$. The timescale for each process is in good agreement with that in the system without TA, indicating that TA does not significantly affect the primary photoreaction of KCD with NPG. 4) The KCD ketyl radical decays by itself and is also scavenged by TA after $~ 930 \mathrm{~ns}$. According to the biexponential fitting of transient absorption at $528 \mathrm{~nm}$, the lifetime of KCD T $\mathrm{T}_{1}$ state and ketyl radical is 0.68 and $7.78 \mu \mathrm{s}$, respectively, for the KCD/NPG "photoinitibitor" 

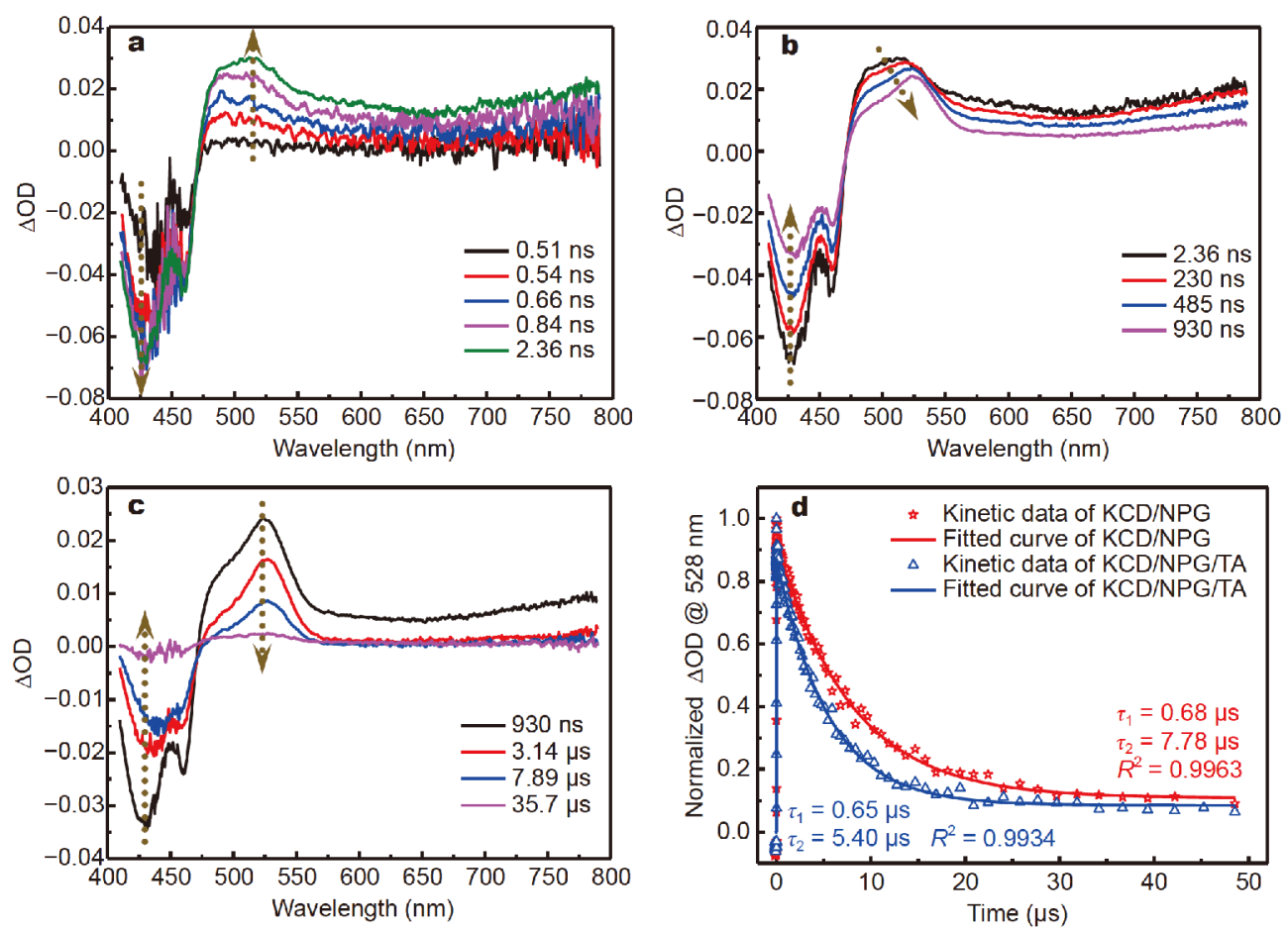

Figure 6 (a-c) Nanosecond transient absorption when irradiating the KCD/NPG/TA mixture $\left(175 \mu\right.$ mol L ${ }^{-1}$ for each in toluene) by a $400 \mathrm{~nm}$ pulse laser. (d) The transitent decay of KCD/NPG and KCD/NPG/TA mixtures at $528 \mathrm{~nm}$. Pseduo-biexponential fitting is implemented to give the lifetime.

(Fig. 6d). By contrast, the lifetime changes to 0.65 and $5.40 \mu$ s when adding TA, respectively, further supporting the interpretation that the addition of TA does not affect the primary photoreaction of KCD with NPG but clearly scavenges the ketyl radical.

To further demonstrate the elimination of ketyl radical by TA, we exerted the EPR characterization upon visible light irradiation. DMPO was also added to capture the radical. Interestingly, distinct from the KCD/NPG "photoinitibitor" (Fig. 4), no signal related to the ketyl radical is detected in the KCD/NPG/TA system (Fig. 7a). On the contrary, a new signal related to the TA derived radical is detected, suggesting that the ketyl radical is scavenged by TA (Fig. 7b). The TA derived radical is believed to be an initiating radical $[38,56]$. The hyperfine coupling constants of radical 3 ( $g$-value, 2.0061) are $20.6 \mathrm{G}(\mathrm{H} 3)$ and $14.3 \mathrm{G}(\mathrm{N} 2)$, in quantitative agreement with those in the KCD/NPG "photoinitibitor". The hyperfine coupling constants of radical 4 ( $g$-value, 2.0062) are $20.1 \mathrm{G}(\mathrm{H} 3)$ and $13.5 \mathrm{G}(\mathrm{N} 2)$, which are also similar to those of radical 3 .

\section{Effect of ketyl radical on the photopolymerization and phase separation}

As shown in Fig. 8, the addition of equivalent TA into the
KCD/NPG mixture significantly increases the polymerization rate and accelerates the gelation process due to the conversion of the inhibiting ketyl radical to a new initiating radical. For instance, the maximum polymerization rate is increased by $86 \%$ (i.e., from 0.086 to $0.160 \mathrm{~s}^{-1}$ ) and the gelation time is decreased by $41 \%$ (i.e., from 34 to $20 \mathrm{~s}$ ).

However, with respect to the fabricated holographic polymer/LC composites, the diffraction efficiency is dramatically decreased from $93 \pm 4 \%$ to $11 \pm 4 \%$ when converting the inhibiting ketyl radical to a new initiating radical (Fig. 9a), primarily because of the reduced diffraction (Fig. S9) caused by the depressed phase separation. As characterized by SEM, the holographic grating ordered structures photo-mediated by the KCD/NPG "photoinitibitor" show a well-defined phase separation with ordered dark holes after removing the LC, whereas the grating ordered structures photo-mediated with the KCD/NPG/TA system exhibit a poor phase separation (Fig. 9b). The AFM characterization also shows that the grating ordered structure depth dramatically decreases from $128.1 \pm 5.9$ to $60.4 \pm 1.9 \mathrm{~nm}$ (Fig. 9c). As a consequence, the former can be electric-switched while the electro-optic performance of the latter is dramatically deteriorated. Holographic images are further re- 
<smiles>CNc1ccccc1</smiles><smiles>CC1=CCCC1(C)C</smiles>

$\mathrm{R}^{\cdot}$<smiles>CC1(C)CC[C@@H](Nc2ccccc2)N1O</smiles>

Radical 3

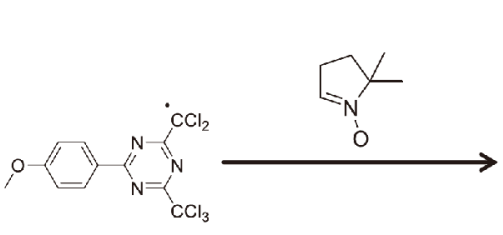

TA

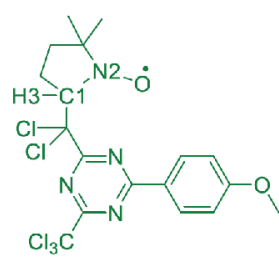

Radical 4
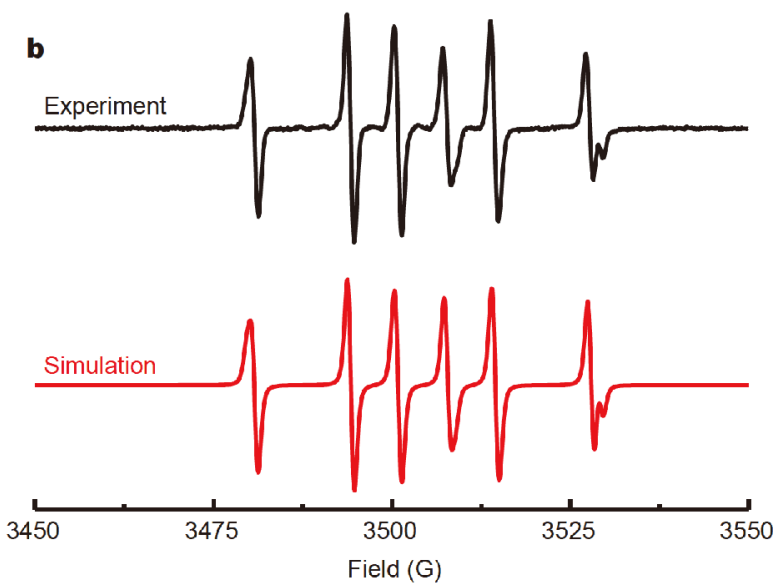

Figure 7 (a) Chemical structures of radicals 3 and 4. (b) Experimental and simulated EPR signals of the KCD/NPG/TA system.
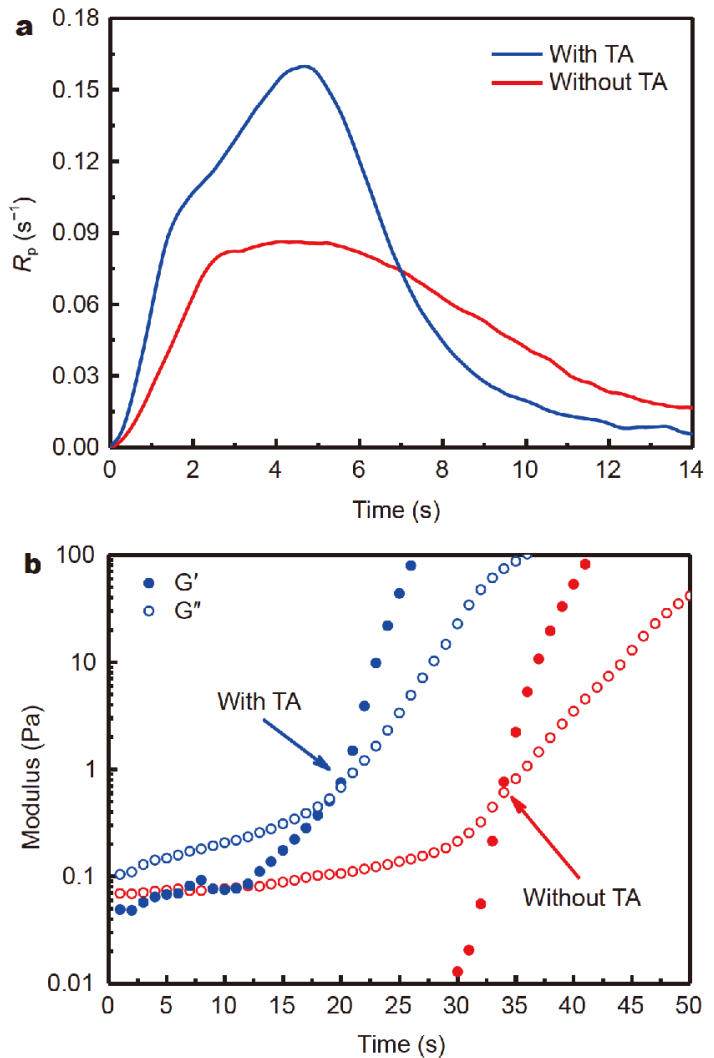

Figure 8 Polymerization rate (a) and modulus (b) of the holographic mixture against the irradiation time upon a $460 \mathrm{~nm}$ light irradiation $\left(3.0 \mathrm{~mW} \mathrm{~cm}^{-2}\right)$. The concentration of $\mathrm{KCD}$ is $6 \mathrm{mmol} \mathrm{L}^{-1}$, while that of NPG and TA are $60 \mathrm{mmol} \mathrm{L}^{-1}$, respectively.
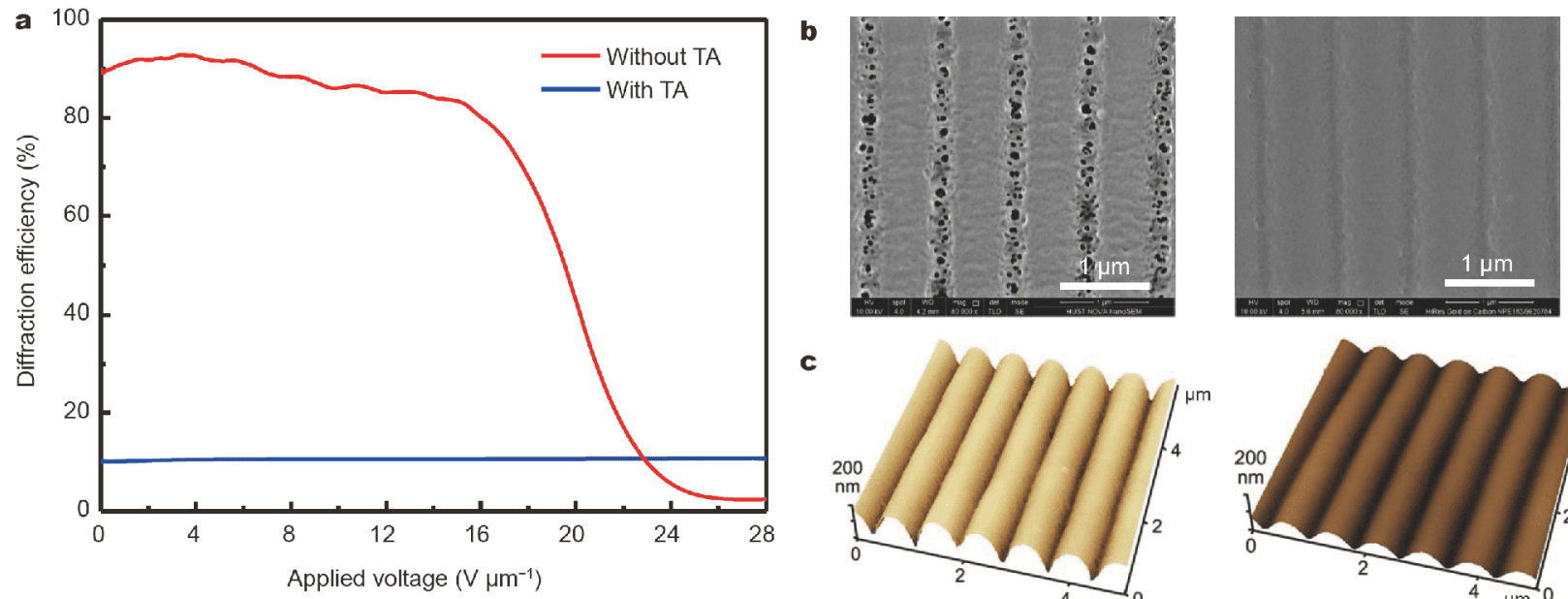

c
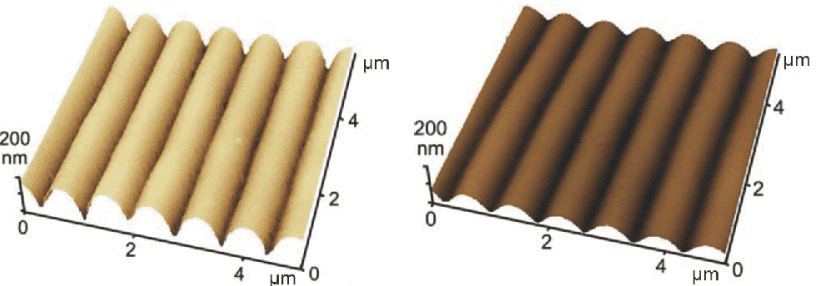

Figure 9 (a) Electro-optic response of holographic polymer composites with the LC fabricated with the KCD/NPG "photoinitibitor" and KCD/NPG/ TA system. SEM (b) and AFM (c) images of the holographic polymer composites with the KCD/NPG "photoinitibitor" (left) and KCD/NPG/TA system (right) after removing the LC. The dark holes in the SEM images and dark channels in the AFM images represent the orginal location of the LC. AFM scanning is hard to give the hole morphology because of the limited tip resolution. The grating ordered structure depth in the AFM images is $128.1 \pm 5.9$ and $60.4 \pm 1.9 \mathrm{~nm}$, respectively. The grating pitch is measured to be $840 \pm 10$ and $830 \pm 40 \mathrm{~nm}$ from the SEM and AFM images, respectively, which is slightly smaller than the predesigned $(889 \mathrm{~nm})$. 
constructed to compare the KCD/NPG "photoinitibitor" and KCD/NPG/TA system (Fig. 10). Clearly, the former is able to generate a much brighter holographic image than the latter. Therefore, in comparison with the KCD/ NPG "photoinitibitor", the KCD/NPG/TA system exhibits a poorer spatiotemporal control over the holographic photopolymerization. We suspect that all radicals tend to diffuse from the constructive regions to the destructive regions because of their own concentration gradients (Scheme 5). Nevertheless, the inhibiting radical has more chance to diffuse than the initiating radicals because of the differences in the concentrations of the vinyl groups versus the radicals. A big difference in polymerization is expected between the constructive and destructive regions when employing the KCD/NPG "photoinitibitor", which leads to a decreased polymerization area. We indeed observed a large gelation time
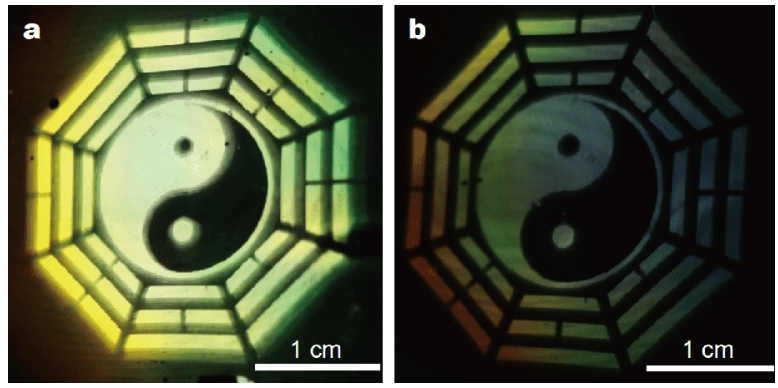

Figure 10 Holographic images reconstructued with the KCD/NPG "photoinitibitor" (a) and KCD/NPG/TA system (b). The images are viewed at the identical angle. difference between these two regions at a high concentration of KCD [13]. By contrast, a proliferated diffusion of initiating radicals in the KCD/NPG/TA system is expected, which leads to an increase of polymerization areas. The increase of polymerization areas is also observed in other systems because of the proliferation of active centers [57].

\section{CONCLUSIONS}

In summary, the KCD/NPG "photoinitibitor" and KCD/ NPG/TA initiating system were compared based on the $\mathrm{UV}$-vis absorption of ground state, transient absorption, EPR spectra, photopolymerization kinetics, photorheology, morphology, electro-optic performance and DFT calculations. The KCD/NPG "photoinitibitor" held the distinct capability of simultaneously generating one initiating radical and one inhibiting ketyl radical. The ketyl radical possessed a long lifetime of $7.78 \mu$ s to inhibit the polymerization via direct radical coupling. The KCD/ NPG "photoinitibitor" was readily converted to an efficient initiating system with depressed inhibition function when adding TA. This conversion was implemented without affecting the primary photoreaction of KCD with NPG. In comparison with the KCD/NPG/TA system, the KCD/NPG "photoinitibitor" afforded an 8.5 times larger diffraction efficiency to the holographic polymer/LC composites because of the improved phase separation by the ketyl radical inhibition.

Received 27 June 2019; accepted 7 August 2019;

published online 2 September 2019

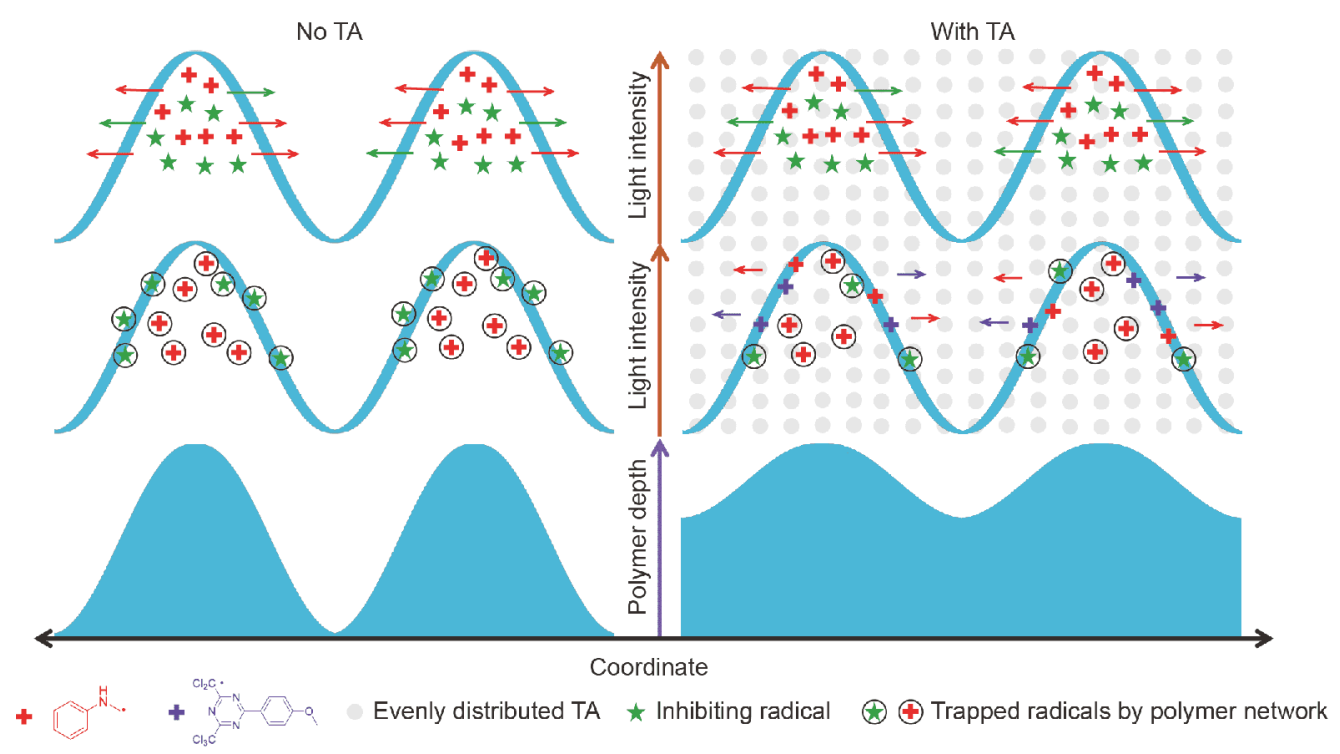

Scheme 5 Proposed radical diffusion during holographic photopolymerization. 
1 Gabor D. A new microscopic principle. Nature, 1948, 161: 777-778

2 Gorkhover T, Ulmer A, Ferguson K, et al. Femtosecond X-ray Fourier holography imaging of free-flying nanoparticles. Nat Photon, 2018, 12: 150-153

3 Tikan A, Bielawski S, Szwaj C, et al. Single-shot measurement of phase and amplitude by using a heterodyne time-lens system and ultrafast digital time-holography. Nat Photon, 2018, 12: 228-234

4 Leite IT, Turtaev S, Jiang X, et al. Three-dimensional holographic optical manipulation through a high-numerical-aperture soft-glass multimode fibre. Nat Photon, 2017, 12: 33-39

5 Vyas S, Chia YH, Luo Y. Conventional volume holography for unconventional airy beam shapes. Opt Express, 2018, 26: 2197921991

6 Melde K, Mark AG, Qiu T, et al. Holograms for acoustics. Nature, 2016, 537: 518-522

7 van den Heuvel M, Prenen AM, Gielen JC, et al. Patterns of diacetylene-containing peptide amphiphiles using polarization holography. J Am Chem Soc, 2009, 131: 15014-15017

8 Kobayashi Y, Abe J. Real-time dynamic hologram of a 3D object with fast photochromic molecules. Adv Opt Mater, 2016, 4: 13541357

9 Blanche PA, Bablumian A, Voorakaranam R, et al. Holographic three-dimensional telepresence using large-area photorefractive polymer. Nature, 2010, 468: 80-83

10 Ozaki M, Kato J, Kawata S. Surface-plasmon holography with white-light illumination. Science, 2011, 332: 218-220

11 Chen G, Ni M, Peng H, et al. Photoinitiation and inhibition under monochromatic green light for storage of colored 3D images in holographic polymer-dispersed liquid crystals. ACS Appl Mater Interfaces, 2017, 9: 1810-1819

12 Xie XL, Peng HY, Zhou XP, et al. Visible Light Photoinitiating System for Preparing High Diffraction Efficiency Hologram Optical Polymer Material. USA Patent, US 9753431 B2, 2017-09-05

13 Peng H, Bi S, Ni M, et al. Monochromatic visible light "photoinitibitor": Janus-faced initiation and inhibition for storage of colored 3D images. J Am Chem Soc, 2014, 136: 8855-8858

14 Ni M, Peng H, Liao Y, et al. 3D image storage in photopolymer/ $\mathrm{ZnS}$ nanocomposites tailored by "photoinitibitor". Macromolecules, 2015, 48: 2958-2966

15 Li X, Ren H, Chen X, et al. Athermally photoreduced graphene oxides for three-dimensional holographic images. Nat Commun, 2015, 6: 6984

16 Luo Y, Gelsinger PJ, Barton JK, et al. Optimization of multiplexed holographic gratings in PQ-PMMA for spectral-spatial imaging filters. Opt Lett, 2008, 33: 566-568

17 Yu R, Li S, Chen G, et al. Monochromatic "photoinitibitor"mediated holographic photopolymer electrolytes for lithium-ion batteries. Adv Sci, 2019, 6: 1900205

18 Shen W, Wang L, Chen G, et al. A facile route towards controllable electric-optical performance of polymer-dispersed liquid crystal via the implantation of liquid crystalline epoxy network in conventional resin. Polymer, 2019, 167: 67-77

19 Shen W, Wang L, Zhong T, et al. Electrically switchable light transmittance of epoxy-mercaptan polymer/nematic liquid crystal composites with controllable microstructures. Polymer, 2019, 160: 53-64

20 Zhao D, Zhou W, Cui X, et al. Alignment of liquid crystals doped with nickel nanoparticles containing different morphologies. Adv Mater, 2011, 23: 5779-5784

21 Hu X, de Haan LT, Khandelwal H, et al. Cell thickness dependence of electrically tunable infrared reflectors based on polymer stabilized cholesteric liquid crystals. Sci China Mater, 2017, 61: 745-751

22 Bunning TJ, Natarajan LV, Tondiglia VP, et al. Holographic polymer-dispersed liquid crystals (H-PDLCs). Annu Rev Mater Sci, 2000, 30: 83-115

23 White TJ, Natarajan LV, Tondiglia VP, et al. Monomer functionality effects in the formation of thiol-ene holographic polymer dispersed liquid crystals. Macromolecules, 2007, 40: 1121-1127

24 Peng H, Yu L, Chen G, et al. Liquid crystalline nanocolloids for the storage of electro-optic responsive images. ACS Appl Mater Interfaces, 2019, 11: 8612-8624

25 Ni M, Chen G, Wang Y, et al. Holographic polymer nanocomposites with ordered structures and improved electro-optical performance by doping POSS. Compos Part B-Eng, 2019, 174: 107045

26 Yagci Y, Jockusch S, Turro NJ. Photoinitiated polymerization: Advances, challenges, and opportunities. Macromolecules, 2010, 43: 6245-6260

27 Dadashi-Silab S, Doran S, Yagci Y. Photoinduced electron transfer reactions for macromolecular syntheses. Chem Rev, 2016, 116: 10212-10275

28 Aguirre-Soto A, Lim CH, Hwang AT, et al. Visible-light organic photocatalysis for latent radical-initiated polymerization via $2 \mathrm{e}^{-} /$ $1 \mathrm{H}^{+}$transfers: Initiation with parallels to photosynthesis. J Am Chem Soc, 2014, 136: 7418-7427

29 Xi W, Pattanayak S, Wang C, et al. Clickable nucleic acids: Sequence-controlled periodic copolymer/oligomer synthesis by orthogonal thiol-X reactions. Angew Chem Int Ed, 2015, 54: 1446214467

30 Zhang J, Xiao P. 3D printing of photopolymers. Polym Chem, 2018, 9: $1530-1540$

31 Michalek L, Barner L, Barner-Kowollik C. Polymer on top: Current limits and future perspectives of quantitatively evaluating surface grafting. Adv Mater, 2018, 30: e1706321

32 Zhang J, Zivic N, Dumur F, et al. N-[2-(dimethylamino)ethyl]-1,8naphthalimide derivatives as photoinitiators under LEDs. Polym Chem, 2018, 9: 994-1003

33 Yu J, Gao Y, Jiang S, et al. Naphthalimide aryl sulfide derivative norrish type I photoinitiators with excellent stability to sunlight under near-UV LED. Macromolecules, 2019, 52: 1707-1717

34 Yang H, Li G, Stansbury JW, et al. Smart antibacterial surface made by photopolymerization. ACS Appl Mater Interfaces, 2016, 8: 28047-28054

35 Deng J, Wang L, Liu L, et al. Developments and new applications of UV-induced surface graft polymerizations. Prog Polymer Sci, 2009, 34: 156-193

36 Zhang L, Du W, Nautiyal A, et al. Recent progress on nanostructured conducting polymers and composites: Synthesis, application and future aspects. Sci China Mater, 2018, 61: 303-352

37 Fouassier JP, Allonas X, Burget D. Photopolymerization reactions under visible lights: Principle, mechanisms and examples of applications. Prog Org Coatings, 2003, 47: 16-36

38 Grotzinger C, Burget D, Jacques P, et al. Photopolymerization reactions initiated by a visible light photoinitiating system: Dye/ amine/bis(trichloromethyl)-substituted-1,3,5-triazine. Macromol Chem Phys, 2001, 202: 3513-3522

39 Peng H, Yu L, Chen G, et al. Low-voltage-driven and highly-diffractive holographic polymer dispersed liquid crystals with spherical morphology. RSC Adv, 2017, 7: 51847-51857

40 Stoll S, Schweiger A. Easyspin, a comprehensive software package 
for spectral simulation and analysis in EPR. J Magn Reson, 2006, 178: 42-55

41 Peng $\mathrm{H}$, Ni M, Bi S, et al. Highly diffractive, reversibly fast responsive gratings formulated through holography. RSC Adv, 2014, 4: $4420-4426$

42 Peng H, Nair DP, Kowalski BA, et al. High performance graded rainbow holograms via two-stage sequential orthogonal thiol-click chemistry. Macromolecules, 2014, 47: 2306-2315

43 Winter HH, Chambon F. Analysis of linear viscoelasticity of a crosslinking polymer at the gel point. J Rheology, 1986, 30: 367382

44 Scott TF, Kowalski BA, Sullivan AC, et al. Two-color single-photon photoinitiation and photoinhibition for subdiffraction photolithography. Science, 2009, 324: 913-917

45 Frisch MJ, Trucks GW, Schlegel HB, et al. Gaussion, D.01. Wallingford CT: Gaussion, Inc. 2013

46 Yamaji M, Oshima J, Hidaka M. Verification of the electron/proton coupled mechanism for phenolic $\mathrm{H}$-atom transfer using a triplet $\pi, \pi^{*}$ carbonyl. Chem Phys Lett, 2009, 475: 235-239

47 Christensen SK, Chiappelli MC, Hayward RC. Gelation of copolymers with pendent benzophenone photo-cross-linkers. Macromolecules, 2012, 45: 5237-5246

48 Li MD, Du Y, Chuang YP, et al. Water concentration dependent photochemistry of ketoprofen in aqueous solutions. Phys Chem Chem Phys, 2010, 12: 4800-4808

49 McIntire GL, Blount HN, Stronks HJ, et al. Spin trapping in electrochemistry. 2. Aqueous and nonaqueous electrochemical characterizations of spin traps. J Phys Chem, 1980, 84: 916-921

50 Odian G. Radical Chain Polymerization. In Principles of Polymerization, 4th ed. Hoboken, New Jersey: John Wiley \& Sons, Inc. 2004. P198-349

51 Church DF. Substituent effects on nitroxide hyperfine splitting constants. J Org Chem, 1986, 51: 1138-1140

52 Sargent FP, Gardy EM. Spin trapping of radicals formed during radiolysis of aqueous solutions. Direct electron spin resonance observations. Can J Chem, 1976, 54: 275-279

53 Ni ML, Peng HY, Xie XL. Structure regulation and performance of holographic polymer dispersed liquid crystals. Acta Polym Sin, 2017, 48: 1557-1573

54 Peng H, Chen G, Ni M, et al. Classical photopolymerization kinetics, exceptional gelation, and improved diffraction efficiency and driving voltage in scaffolding morphological H-PDLCs afforded using a photoinitibitor. Polym Chem, 2015, 6: 8259-8269

$55 \mathrm{Ni}$ M, Chen G, Sun H, et al. Well-structured holographic polymer dispersed liquid crystals by employing acrylamide and doping $\mathrm{ZnS}$ nanoparticles. Mater Chem Front, 2017, 1: 294-303

56 Kabatc J, Czech Z, Kowalczyk A. The application of halomethyl 1,3,5-triazine as a photoinitiator or co-initiator for acrylate monomer polymerization. J Photochem Photobiol A-Chem, 2011, 219: $16-25$

57 He M, Huang X, Zeng Z, et al. Phototriggered base proliferation: A highly efficient domino reaction for creating functionally photoscreened materials. Macromolecules, 2013, 46: 6402-6407

Acknowledgements We thank the financial supports from the National Natural Science Foundation of China (51433002 and 51773073), HUST peak boarding program, the National Science Foundation (NSF) of Hubei Scientific Committee (2016CFA001) and the Fundamental Research Funds for the Central Universities (2019kfyRCPY089). We also thank the technical assistance from HUST Analytical \& Testing Center.
Author contributions Li MD, Xie X and Peng $\mathrm{H}$ gave the direction of the experiments; Zhao $\mathrm{X}$ and Sun $\mathrm{S}$ conducted the experiments together; Zhao Y participated in the discussion; Liao RZ obtained the DFT calculation results; Liao $\mathrm{Y}$ gave suggestions to the experiments and revised the manuscript with Peng $\mathrm{H}$.

Conflict of interest The authors declare no competing financial interest.

Supplementary information Supporting data are available in the online version of the paper.

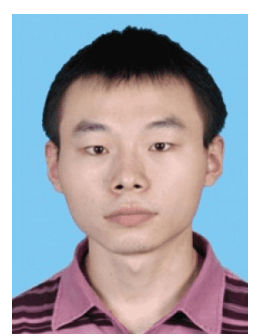

Xiaoyu Zhao received his Master's degree from Shanghai Institute of Organic Chemistry, Chinese Academy of Sciences in 2016. He is now a $\mathrm{PhD}$ candidate at Huazhong University of Science and Technology (HUST) under the supervision of Prof. Xiaolin Xie and Prof. Haiyan Peng. His current interest focuses on photopolymerization mechanism and applications.

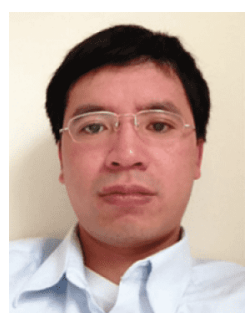

Ming-De Li obtained his PhD degree at the University of Hong Kong in 2012. Then he conducted his postdoctoral research at the University of California, Berkeley and The University of Hong Kong. Now, he is a professor at Shantou University. His current interest is in the ultrafast laser spectroscopies.

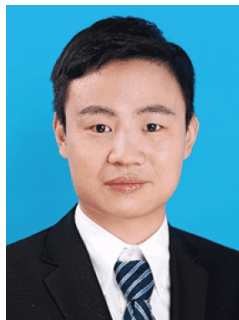

Haiyan Peng received his $\mathrm{PhD}$ degree from HUST in 2014. He visited the University of Colorado Boulder from 2012 to 2014, sponsored by the CSC. Then he did research as an Assistant Professor at Guangzhou Institute of Advanced Technology, Chinese Academy of Sciences, and conducted postdoctoral research at City University of Hong Kong. He has been an Associate Professor at HUST since 2016.

\section{羰基自由基对全息聚合物/液晶复合材料结构及 性能的影响}

赵骁宇 ${ }^{1}$, 孙姗姗 ${ }^{2}$, 赵晔 ${ }^{1}$, 廖荣榛 ${ }^{1}$, 李明德 ${ }^{2 *}$, 廖永贵 ${ }^{1}$, 彭海炎 ${ }^{1^{*}}$, 解孝林 ${ }^{1}$

摘要 全息聚合物/液晶复合材料是由富聚合物相与富液晶相周期 性排列而成的结构有序复合材料, 不仅具有独特的彩色 $3 \mathrm{D}$ 图像存 储功能, 还具有电光响应特性, 因此获得了广泛关注. 全息聚合物/ 液晶复合材料通过激光相干下的光聚合诱导相分离原位形成. 高 的光聚合反应速率有利于全息加工，但往往会抑制相分离. 因此, 发展新的动力学调控策略以提升全息聚合物/液晶复合材料的性能 仍是一个挑战. 本研究发现, 羰基自由基阻聚可抑制引发自由基从 相干亮区向相干暗区的传递，进而显著提高相分离程度和全息性 能. 消除羰基自由基导致全息聚合物/液晶复合材料性能下降，也 证实了羰基自由基阻聚在设计高性能全息聚合物/液晶复合材料中 的重要性. 